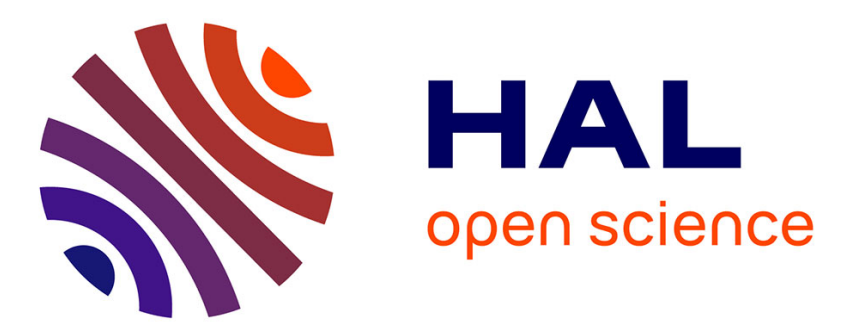

\title{
Infrared-Thermodynamics Conversion as a Function of Temperature: Towards Confined Water
}

\author{
Isabelle Bergonzi, Lionel Mercury
}

\section{To cite this version:}

Isabelle Bergonzi, Lionel Mercury. Infrared-Thermodynamics Conversion as a Function of Temperature: Towards Confined Water. Mercury, Lionel; Tas, Niels; Zilberbrand, Michael (Eds.). Transport and Reactivity of Solutions in Confined Hydrosystems, Springer, pp.43-53, 2013, Series: NATO Science for Peace and Security Series C: Environmental Security, 10.1007/978-94-007-7534-3_4 . insu00906892

\section{HAL Id: insu-00906892 \\ https://hal-insu.archives-ouvertes.fr/insu-00906892}

Submitted on 20 Nov 2013

HAL is a multi-disciplinary open access archive for the deposit and dissemination of scientific research documents, whether they are published or not. The documents may come from teaching and research institutions in France or abroad, or from public or private research centers.
L'archive ouverte pluridisciplinaire HAL, est destinée au dépôt et à la diffusion de documents scientifiques de niveau recherche, publiés ou non, émanant des établissements d'enseignement et de recherche français ou étrangers, des laboratoires publics ou privés. 
Infrared-Thermodynamics Conversion as a Function of Temperature: Towards Confined Water

Isabelle Bergonzi and Lionel Mercury

Institut des Sciences de la Terre d'Orléans, UMR 7327 Université d'Orléans-CNRS-BRGM, 1A rue de la Férollerie, 45071 Orléans Cedex, France

\begin{abstract}
An experimental method has been developed to calculate the thermodynamic properties of water from its vibrational properties, relevant to study (in near future) the properties of adsorbed or confined water. The infrared absorption of the intra-molecular $\mathrm{OH}$ stretching mode of liquid water has been measured over a wide range of temperature (from $-10^{\circ} \mathrm{C}$ to $90^{\circ} \mathrm{C}$ ). The corresponding large band has been decomposed into three Gaussian components standing for three different water connectivities (percolation model) that feature the liquid state as a function of temperature: network, intermediate, multimer, water. Measurements evidenced that the components are differently shifted with temperature, giving a quantitative insights into the internal energy change of liquid. A vibrational partition function has been used to calculate the corresponding thermodynamic properties, neglecting all energy components except the present intra-molecular vibrational mode. Interestingly, the vibrational free enthalpy thus computed differs of the total free enthalpy only by a multiplicative constant all along the thermal range.
\end{abstract}

\title{
1. INTRODUCTION
}

Liquid water presents a remarkable ability to fill in any cavity or channel, even of nanometric dimensions (confinement), and to stay as thin films onto almost any solid surfaces (adsorption). The term confinement relates to the influence of the close solids on the sandwiched liquid, while adsorption is a confinement between solid and air. Interestingly, confinement and adsorption are states that allow liquid water to stay inside a pore network under arid or semi-arid conditions, affording a minimum water content in soils, with the thin films hydraulically connecting capillary clusters confined in wedges, crevices or small channels. This minimum water content directly impacts the soil wettability, its ability to host drought-tolerant species, and to maintain residual microbial activity and mobility. In terms of weathering, these water types are intimately contacting the host solid and also stay immobile for long period of time, making them a probably important (and overlooked) actor in the global mass balance.

Confined and adsorbed waters have properties that deviate from the bulk water (e.g., [1-7]), which give them specific driving forces in terms of transport, weathering, and biological habitats. Confined water is found in saturated and nonsaturated media, actually whenever liquid is occluded in host spaces smaller than $0.1 \mu \mathrm{m}$ (e.g., [8-12]). Adsorbed water is encountered in non-saturated media, as thin films of nanometer to hundreds of nanometers thickness (e.g., [1,13]). Despite this large versatility in natural settings, the properties of these water types and the characteristic thresholds describing their evolution with sizes/thicknesses are not well apprehended in the natural sciences community. Meanwhile, their characteristic properties are essentially measured at very high confinement around the nanometer, and their thermodynamic behaviour in the $5-100 \mathrm{~nm}$ range is poorly known.

Infrared (IR) spectroscopy have been used to probe the water liquid state with solutes addition [14], when adsorbed on surfaces [11,15] or filling restricted spaces [11,12], at the solid-water or liquid-air interfaces [16]. These studies demonstrated the sensitivity of the IR signal to record slight changes of the properties of liquid, even for small amount of matter. This sensitivity indicates that vibrational energy changes correctly probe energetic changes with environment. Among all the IR absorption modes, the intra-molecular OH-stretching band revealed to be the most significant, showing shifts both in terms of global shape, intensity and location of the main peak. However, there is not a direct conversion of these IR shifts into macroscopic thermodynamic properties to date. Some studies proposed a statistical deconvolution of the IR and Raman spectra to obtain thermodynamic values of diluted solutions of HOD molecules in $\mathrm{D}_{2} \mathrm{O}$ (or $\mathrm{H}_{2} \mathrm{O}$ ), but this approach cannot describe the IR spectra of normal water [17-19].

It is why infrared spectroscopic measurements have been targeted to record the change in the vibrational properties of water trapped into artificial rectangular channels having depths varying from 100 to $5 \mathrm{~nm}$ (1D nano-channels). However, this paper lets aside the confinement topic to deal with a required prior step: devising a calculation method for the IR-thermodynamics conversion based on a partition function, and calibrating it on a well-known thermodynamic change, here the thermodynamic behavior of liquid water from $-10^{\circ} \mathrm{C}$ to $90^{\circ} \mathrm{C}$.

\section{EXPERIMENTAL SECTION}


IR measurements were recorded in the $400-4000 \mathrm{~cm}^{-1}$ spectral region with a Fourier Transform Infra-Red (FTIR) micro-spectrometer (Nicolet Continu $\mu \mathrm{m}$, Thermo scientific), equipped with a XT-KBr beam splitter and a liquid nitrogen cooled Mercury Cadmium Telluride (MCT) detector $(250 \mu \mathrm{m})$. The microscope operates in confocal mode, using a 15x infinity corrected Schwarzchild objective and a matching $15 x$ condenser.

To avoid saturating the IR absorption signal due to the enormous amount of water molecules per liquid volume, a thin film cell of varying thickness, down to micrometer, has been developed. This cell is composed of two steel parts compressing two $\mathrm{BaF}_{2}$ windows $(13 \mathrm{~mm} \times 2 \mathrm{~mm}$ ), sealed with a fluorocarbon O-ring. A liquid water drop is sandwiched between the two windows and the steel parts are tightened to form a bulk water film roughly $1 \mu \mathrm{m}$ thick.

The thermal change is controlled by a heating-cooling stage (THMS-600, Linkam) with windows transparent to IR light. Briefly, the fluid cell is put on a silver block at controlled temperature $\left( \pm 0.1{ }^{\circ} \mathrm{C}\right)$, and the acquisition starts after 5 minutes, which is the time needed to afford thermal homogeneity between the two steel parts.

Each spectrum is recorded with a resolution of $4 \mathrm{~cm}^{-1}$ with 200 scans per spectrum and no mathematical correction. The infrared absorption coefficient is defined by:

$A=-\log \left(I_{0} /{ }_{I}\right)$

where $I_{0}$ is the transmitted intensity of the empty cell and $I$ the transmitted intensity of the cell filled with water. Spectra are recorded from $-10^{\circ} \mathrm{C}$ to $90^{\circ} \mathrm{C}$.

\section{EXPERIMENTAL RESULTS AND DISCUSSION}

\subsection{IR absorption band}

The infrared spectrum of liquid water consists of four main bands, spreading from a few wavenumbers and $3800 \mathrm{~cm}^{-1}$ [20]. The dominating feature of the IR water spectrum is the band located at $3400 \mathrm{~cm}^{-1}$ which corresponds to the stretching motion of the covalent $\mathrm{OH}$ bonds. The $\mathrm{HOH}$ bending band $\left(1650 \mathrm{~cm}^{-1}\right)$ follows in the decreasing frequency order, then the libration band $\left(675 \mathrm{~cm}^{-1}\right)$, and finally the "connectivity" band $\left(200 \mathrm{~cm}^{-1}\right)$. The two former bands correspond to intra-molecular modes, either sensitive to the H-bonds environment (stretching) or not sensitive (bending). The two latter reflect inter-molecular modes. The libration mode, through small amplitude oscillations of the whole molecule, provides a mechanism of rupture and rearrangement of the hydrogen bonds at a sub-picosecond rate. The connectivity band arises from the longitudinal motion of the $\mathrm{H}$-atom along the $\mathrm{H}$-bond axis (H-bond stretching) and therefore characterizes the level of H-bonding between neighboring water molecules.

\section{$3.2 \mathrm{OH}$ stretching band}

The $\mathrm{OH}$ stretching band centred at about $3400 \mathrm{~cm}^{-1}$ is a sensitive probe of the $\mathrm{H}$-bond: the wavenumber increases with the distance between $\mathrm{O}$ atoms of two molecules linked by H-bond (e.g., [21]). The absorption corresponds to intramolecular motions of the water molecules crossed by the IR beam, which depend on the molecular environment. As a result, this is a molecular signal averaged over macroscopic amount of matter. The width of the band echoes the energetic distribution of $\mathrm{H}$-bonds along the beam pathway and then is an image of the molecular disorder, what justifies the intense study of this band (e.g., [22-24]).

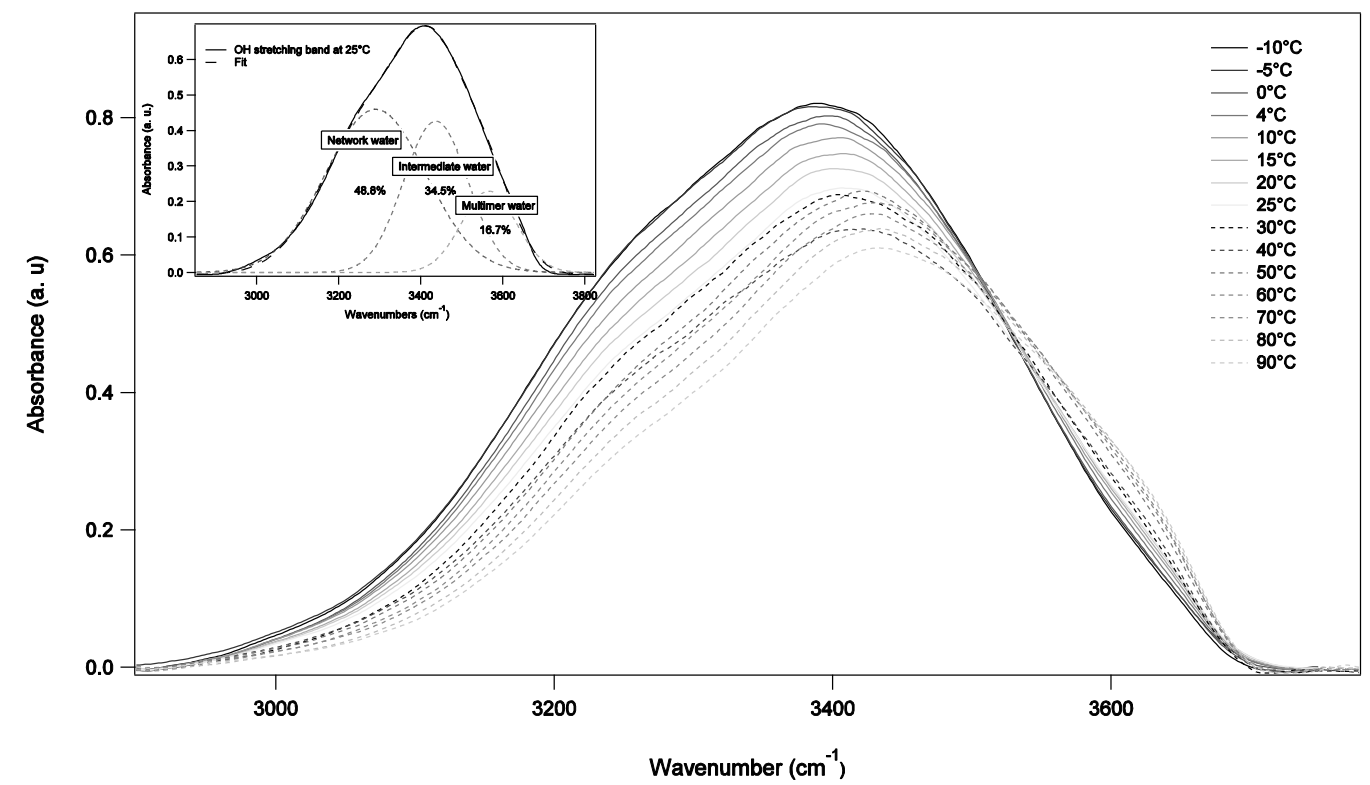

Figure 1: $\mathrm{OH}$ stretching band of liquid water as a function of the temperature. Inset displays the 3 Gaussian components decomposition classically performed (see text). 
The present measurements from $-10^{\circ} \mathrm{C}$ (supercooled water) to $90^{\circ} \mathrm{C}$ (Fig. 1) are in agreement with previous studies [2527]: Upon heating, the main peak moves to higher wavenumbers (blue-shifted, from $3380.6 \mathrm{~cm}^{-1}$ at $-10^{\circ} \mathrm{C}$ up to 3436.5 $\mathrm{cm}^{-1}$ at $90^{\circ} \mathrm{C}$ ), while the intensity diminishes and the shape visually changes losing the lower wavenumbers and enriched on the higher ones. This shift is classically interpreted by the direct effect of heating that disrupts water intermolecular network and then promotes disorder (e.g., [22,23]). This interpretation applies as well to account for the shape change under heating. The decreasing absorbance is readily accounted for, outlining that the resonance condition required for coupling the $\mathrm{OH}$ stretching motion is obtained at low temperature [28].

These visual observations cannot be turn easily into quantitative calculations because liquid water is a continuum of molecular configurations, each having its own behavior and influence on the change of the total energy. And the modeling of the whole band in terms of discrete molecular energies cannot be performed. The common strategy is to decompose the $\mathrm{OH}$-stretching band into Gaussian components, each standing for a certain type of water that features the liquid at a given time. Decomposition into two (e.g., [29]), three (e.g, [20]) and four components (e.g., [14]) have been performed, most often related to the purposely existing water clusters. Actually, the vibrational mode causing the stretching motion of the intra-molecular $\mathrm{OH}$ bond is sensitive to the strength of $\mathrm{H}$-bonding between molecules. The oscillator strength of a water molecule decrease when its local coordination increases and the corresponding $\mathrm{OH}$ frequency is downshifted. The notion of water cluster, and its relation to water structure, is problematic since it is well known that the relaxation time of water is around the picosecond, several order of magnitude quicker than the recording time of the IR absorption. It is why this classic approach has been recently reformulated in terms of the percolation model [30], each Gaussian component featuring an averaged coordination numbers, probabilistically ranging from 0 to 4 [12,20]. The best and most relevant decomposition is done through three Gaussian components (inset in Fig. 1), ascribed to three different types of water molecules. The low-frequency Gaussian is associated to the fully coordinated water molecules, called Network Water (NW) also known as ice-like water since the Gaussian peak is close to the ice's $\left(3250 \mathrm{~cm}^{-1}\right.$, [24]). The high-frequency Gaussian is ascribed to the molecules with a low coordination number, called Multimer Water (MW) and close to the IR characteristics of vapour molecules. The Gaussian lying in-between has a coordination number close to two, is called Intermediate Water (MW) and refers to the incompletely coordinated liquid molecules. At the scale of one molecule, it is clear that each molecule experiences the three coordination states over the experimental time (minute). The final band represents how the molecular network promotes the different types of coordination related to the energy available in the system.

\subsection{Band decomposition}

The experimental bands were fitted with the software Igor and its Multipeak fitting 2.0 package. Brubach et al [20] suggested that the three Gaussian components were linked to the five species described by the percolation model [30]. This model calculates the variation of the coordination number of one water molecules with its immediate neighbors, with temperature. The number of intact $\mathrm{H}$-bonds per molecule is given by the following probability:

$p_{B}=n_{H B} / Z$

with $n_{H B}$ the number of established H-bonds and $z=4$ the maximum number of hydrogen atoms bonded to one oxygen atom (by covalent or intermolecular bond).

Molecules are thus divided into five species, by the possible number of intact H-bonds they form with their neighbors, from 0 to 4 . The fraction $f_{j}$ of water molecule establishing $j$ bonds is given by the relation:

$f_{j}=\frac{z !}{j !(z-j) !} p_{B}^{j}\left(1-p_{B}\right)^{z-j}$

with $j$ ranging from 0 to 4 . The fraction of species $j$ is a function of the temperature $T$ via the temperature dependence of $p_{B}$ written $p_{B}=1.8-0.004 T(K)$.

Brubach et al. [20] proposed to ascribe NW to species $j=4$ and $j=3$, IW to species $j=2$ and MW to species $j=1$ and $j=0$. The interesting point of such interpretation is the implicit use of the ergodic principle to describe the thermodynamic features of liquid water: the IR signal averaged over time echoes the averaged energetic states making up this liquid. Practically, fits were constrained to make the full width at half maximum of the calculated Gaussian component to match the fraction of water molecules "types" calculated by equation (3) with temperature (Fig. 2). Over $-10^{\circ} \mathrm{C}$ to $90^{\circ} \mathrm{C}$, $\mathrm{NW}$ fraction dominates up to $40^{\circ} \mathrm{C}$, from which temperature IW starts predominating. Interestingly, IW is the fraction that varies the less over the thermal range, while the two others are anti-correlated in the expected way: NW decreases with temperature while the MW increases, in agreement with the chaotropic trend afforded by heating individual molecules that tend toward a vapour-like network organization. This variation is in good agreement with the shape evolution of the $\mathrm{OH}$ band with temperature: decreasing absorbance at low wavenumbers echoes the diminution of NW molecular fraction while increasing absorbance at high wavenumbers is naturally linked to the increasing MW molecular fraction. Gaussian maximum are shifted toward lower frequencies with temperature (Fig. 2): NW main peak moves from $3315 \mathrm{~cm}^{-1}$ at $-10^{\circ} \mathrm{C}$ to $3219 \mathrm{~cm}^{-1}$ at $90^{\circ} \mathrm{c}$, IW peak is displaced from $3455 \mathrm{~cm}^{-1}$ at $-10^{\circ} \mathrm{C}$ to $3384 \mathrm{~cm}^{-1}$ at 
$90^{\circ} \mathrm{C}$ and $\mathrm{MW}$ peak slides from $3583 \mathrm{~cm}^{-1}$ at $-10^{\circ} \mathrm{C}$ to $3528 \mathrm{~cm}^{-1}$ at $90^{\circ} \mathrm{C}$. This IR downshifting evidences that the characteristic H-bond lengths of each water "types" decreases, what means that the energy to maintain the corresponding network organization must increase due to the additional thermal energy in the system. Otherwise, to keep its connectivity level, each water "types" uses more energy.

As a consequence, the $\mathrm{OH}$-stretching band highlights that the IR shift is a direct measure of the internal energy change of the probed liquid. Summing up the three contributions, should result in deducing the whole thermodynamic change from a given reference state. In all that follows, the reference state is the pure liquid at $25^{\circ} \mathrm{C}$ and $0.1 \mathrm{MPa}$.

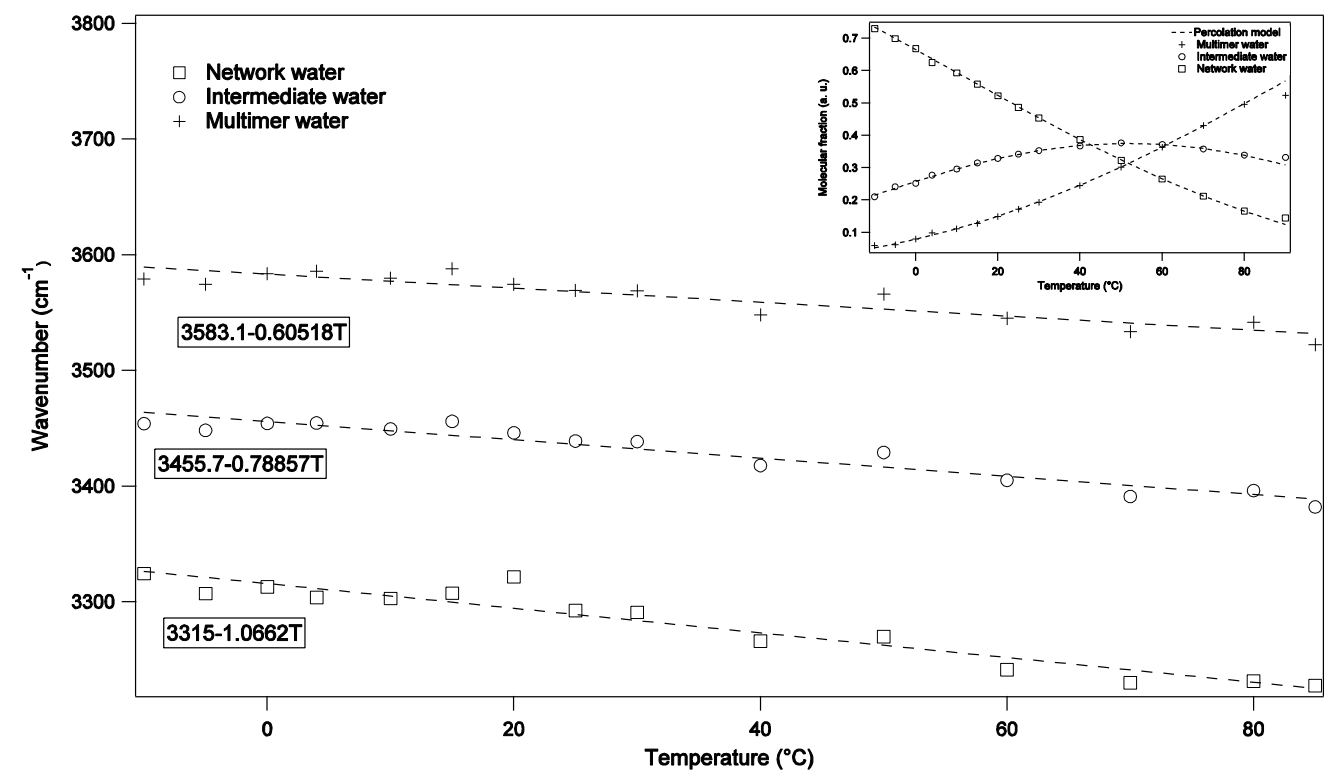

Figure 2: Location of the main peak of the Gaussian components with temperature. The inset shows the evolution of the molecular fraction of the three water populations: NW, IW, MW (see text and eq. (3)).

\subsection{Thermodynamic treatment}

The conversion of the IR absorption band into thermodynamic terms requires a description of the way the characteristics of each energetic state combine into the macroscopic value of the thermodynamic property. The relevant tool is the partition function, here that proposed by Vand and Senior [31]. It is based on the Némethy and Scheraga model [32], which is a continuum model based on the concept of "flickering clusters". The H-bonds formation in liquid water is described as a cooperative process, i.e. H-bonds are established or broken simultaneously depending on the local energy fluctuations. Short-lived regions of highly H-bonded clusters thus take place surrounded by non H-bonded molecules. As a consequence, the model features liquid with two main structures, clusters and non H-bonded molecules. The water clusters can be monomer (one bond), dimer (two bonds), trimer (three bonds), or tetramers (four bonds). Despite this "water structure" background, the partition function is built through five species distinguished by their coordination number (four water clusters and the non H-bonded molecules) and an energy level is ascribed to each species, in an analytical approach completely compatible with the percolation model. The molecules distribution between the energy levels is determined by the corresponding Boltzmann factors, $\exp \left(E_{i} / k T\right)$ where $E_{i}$ is the energy of a given energy level and $k$ is the Boltzmann constant, and by the degrees of freedom allowed for the motion of each species. The corresponding partition function $Z$ writes as follows [32]:

$Z=\sum_{\left[n_{0}, y_{1}, x_{u}\right]} g\left(n_{0}, y_{1}, x_{u}\right) \prod_{i=u}^{i=4}\left[f_{i} \exp \left(-\frac{E_{i}}{R T}\right)\right]^{N_{0} x_{i}}$

where $g$ is a combinatorial factor written $g\left(n_{0}, y_{1}, x_{u}\right)=N_{0} ! /\left(N_{4} ! N_{3} ! N_{2} ! N_{1} ! N_{u} !\right), N_{i}=N_{0} x_{i}$ is the number of molecules of each species, $N_{0}$ is the Avogadro's number, $x_{i}$ are mole fractions of each species in the bulk liquid, $E_{i}$ are the energies of each species and $f_{i}$ are the weighting factors describing the vibrational, rotational and translational freedom allowed to each species, ascribed from IR and Raman experimental data.

The present approach by IR-shift relative to a reference enables to neglect the modes that are not sensitive to the varying parameter (temperature or confinement, or any other). The intra-molecular IR changes are entirely featured by the OH-stretching band, since the bending mode has a negligible thermal coefficient $\left(-0.1 \mathrm{~cm}^{-1} \cdot \mathrm{K}^{-1}\right.$; [33]). The intermolecular modes $\left(60 \mathrm{~cm}^{-1}\right.$ to $1000 \mathrm{~cm}^{-1}$; libration and connectivity bands) include as well the cooperativity between vibration and rotation modes, and both change with temperature (e.g., [20]). Nemethy and Scheraga [32] hypothesized that these intermolecular bands that appear in ice, express the contribution of the clustered water. With their partition function, and experimental values for the IR bands, they were able to accurately re-calculate the entropy, free energy and internal energy of liquid water but failed with the specific heat of water. Based on experimental measurements [34- 
35], Vand and Senior [31] alternatively proposed to describe the partition function with three species, each having a certain distribution around a mean effective length $\bar{l}$ of H-bond in liquid water. As a consequence, the energy of molecules in an energy band (mean effective length $\bar{l}$ ) will have a similar regular distribution about a mean value $E_{i}$. Vand and Senior [31] selected arbitrarily a Gaussian distribution to describe the distribution of the three energy bands, and the partition function becomes:

$Z=\sum_{i=1}^{3} \frac{g_{i} X^{m_{i}}}{\Delta_{i}} \exp \left\{\frac{1}{4}\left(\Delta_{i}^{2} X^{2}\right)-\bar{E}_{i}\right\}$

where $X=1 / R T, E_{i}$ is the energy of the ith species, $g_{i} X^{m_{i}}$ is the power law expression for the weighting factor $f_{i}$ that integrate thermal variation, $\Delta$ is the bandwidth of the energy distribution and $B$ is a constant.

At first sight, the partition function with three energy bands fits well the decomposition into three Gaussian components done from the $\mathrm{OH}$-stretching band. Using the Gaussian changes as a function of temperature, the Vand and Senior function offers a simple pathway to transform IR shifts into macroscopic water energy changes. In liquid water, the bending and $\mathrm{OH}$-stretching bands are intra-molecular modes and only the latter moves with the temperature. In the meantime, there are frustrated (with respect to vapor) rotational motions (librations, around $675 \mathrm{~cm}^{-1}$ ) due to the networking that changes the inter-molecular vibrational mode of $\mathrm{H}$-atoms. A second inter-molecular mode is featured by the hindered translational (or connectivity) band (around $200 \mathrm{~cm}^{-1}$ ), which correspond to the longitudinal motion of the $\mathrm{H}$-atom along the $\mathrm{H}$-bond axis (H-bond stretching) and therefore characterizes the level of $\mathrm{H}$ bonding between neighboring water molecules. Among these four bands, only two exhibit pronounced substructures that significantly evolve with temperature (and probably confinement) (e.g, $[12,20])$ : the OH-stretching band and the connectivity bands. These substructures characterize the changing levels of $\mathrm{H}$ bonding in the molecular network that are directly related to the thermodynamic properties. As a consequence, the coming interpretation is weakened by the sole consideration of the stretching band, while (at least) the connectivity band contributes to the internal energy.

The IR-thermodynamics conversion corresponds to transform the total mean energies value in equation (5) [31] into the three vibrational mean energies given by the peak of the three Gaussian components, resulting in the "vibrational" thermodynamic functions of liquid water as a function of temperature (Fig. 3A). Consistently, the vibrational Gibbs free energy is smaller than the total Gibbs free energy ignoring the contribution of the inter-molecular modes.
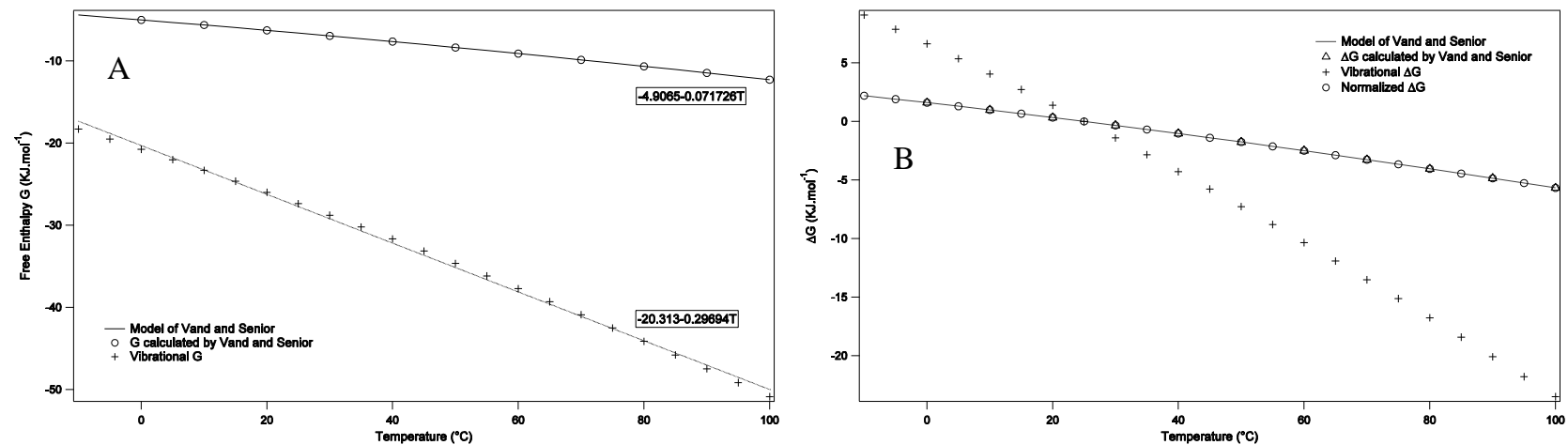

Figure 3. A. Evolution of the total and vibrational free enthalpies (or Gibbs free energies) as a function of temperature. Dotted line is only guide for eyes. B. Evolution of vibrational and total free enthalpies relative to $25^{\circ} \mathrm{C}$. The solid line (calculated Gibbs free energy) is perfectly fitted by the vibrational Gibbs free energy (open circles) multiplied by the constant C $(C=0.241546$, see text $)$.

This first absolute calculation can be easily normalized at $25^{\circ} \mathrm{C}$, and only the relative change to this reference is calculated (Fig. 3B), removing all the modes that are not sensitive to thermal change. Here again, $\Delta \mathrm{G}_{\mathrm{vib}}$ has a larger slope than total $\Delta \mathrm{G}$, outlining that the intra-molecular vibrational mode is compensated by another energetic contribution of opposite trend with temperature. Consistently, rotational and/or translational motions tend to increase with increasing temperature due to the decreasing of the coordination number of water molecules that favors non $\mathrm{H}$ bond molecules. Assuming that the frustrated rotation (libration) does not change with temperature, and that any other rotation and/or translation modes impact the total energy, we may propose that a simple subtraction lead to predict the change of the inter-molecular connectivity mode. At this stage, obviously, an experimental control of such statement must be done.

For the time being, the multiplicative constant $\mathrm{C}$ that enables to retrieve the total $\Delta \mathrm{G}$ (i.e. $\Delta G_{\text {total }}=C \times \Delta G_{v i b}$;) all along the thermal range demonstrates that the thermodynamic changes under temperature, are as linear as the intramolecular $\mathrm{OH}$-stretching mode. Thus, the decomposition of the $\mathrm{OH}$ stretching band into three Gaussian components, coupled with the Vand and Senior partition function, allows us to obtain a thermodynamic description, though incomplete, of water. Technically, it is interesting to outline the compatibility between the percolation model and the 
original Nemethy and Scheraga approach. The probabilistic H-bonds networking can be assumed in the same continuum framework as that afforded by the flickering water-structured clusters.

\section{CONCLUSION}

The IR-to-bulk thermodynamics conversion has been explored following the OH-stretching band behaviour with temperature. The method, based on the percolation model and using mechanistic partition function, appears reliable and gives the correct trend. However, it fails to become strictly quantitative, most probably by overlooking the other significant contribution, mainly the connectivity band. These effects need now to be evaluated with temperature, to decipher the missing link between $\Delta G_{v i b}$ and $\Delta G_{\text {total }}$.

These results give confidence about the ability of the FTIR spectroscopy to probe the thermodynamic properties of liquid water under given conditions. Thus, the protocols to record IR absorption under confinement have been developed as well as the design of the artificial channels with varying depth (1D nano-confinement).

\section{ACKNOWLEDGMENTS}

This work has received support from the French Agency for Research (Agence Nationale de la Recherche, ANR) through the grant CONGE BLAN-61001 and ANR-10-LABX-100-01.

\section{REFERENCES}

[1] Derjaguin BV, Churaev NV and Muller VM (1987) Surface forces. Plenum Publishing Corporation, New York

[2] Handa YP, Zakrzewski M, and Fairbridge C (1992) Effect of restricted geometries on the structure and thermodynamic properties of ice. J Phys Chem 96: 8594-8599

[3] Teixeira J, Zanotti J-M, Bellissent-Funel M-C and Chen S-H (1997) Water in confined geometries. Physica B 234: 370-374

[4] Dore J (2000) Structural studies of water in confined geometry by neutron diffraction. Chem Phys 258: 327-347

[5] Christenson HK (2001) Confinement effects on freezing and melting. J Phys: Cond Matter 13: 95-133

[6] Johari GP (2005) Water's size-dependent freezing to cubic ice. J Chem Phys 122: art. 194504, 5 p

[7] Tombari E, Salvetti G, Ferrari C and Johari GP (2005) Thermodynamic functions of water and ice confined to $2 \mathrm{~nm}$ radius pores. J Chem Phys 122: art. 104712, 9p

[8] Shelton DP (2000) Collective molecular rotation in water and other simple liquids. Chem Phys Lett 325: $513-516$

[9] Putnis A and Mauthe G (2001) The effect of pore size on cementation in porous rocks. Geofluids 1: 37-41

[10] Rijniers LA, Huinink HP, Pel L, and Kopinga K (2005) Experimental evidence of crydtallization pressure inside porous media. Phys Rev Lett 94: 075503

[11] Musat R, Renault JP, Candelaresi M, Palmer DJ, Le Caer S, Righini P, and Pommeret S (2008) Finite size effects on hydrogen bonds in confined water. Angew Chem Int Ed Engl 47: 8033-8035

[12] Le Caër S, Pin S, Esnouf S, Raffy Q, Renault JP, Brubach JB, Greff G and Roy P (2011) A trapped water network in nanoporous material : the role of interfaces. Phys Chem Chem Phys 13: 17658-17666

[13] Pashley RM and Kitchener JA (1979) Surface forces in adsorbed multilayers of water on quartz. J Colloid Interf Sci 71(3): 491-500

[14] Masuda K, Haramaki T, Nakashima S, Habert B, Martinez I, and Kashiwabara S (2003) Structural change of water whith solutes and temperature up to $100^{\circ} \mathrm{C}$ in aqueous solutions as revealed by Attenuated Total Refelctance Infrared Spectroscopy. Appl Spectros 57(3): 274-281

[15] Richard T, Mercury L, Poulet F and d'Hendecourt L (2006) Diffuse reflectance infrared Fourier transform spectroscopy as a tool to characterize water in adsorption/ confinement situations. J Colloid Interf Sci 304: 125-136

[16] Mercury L, Jamme F et Dumas P (2012) Infrared imaging of bulk water and water-solid interface under stable and metastable conditions. Phys Chem Chem Phys 14: 2864-2874

[17] Efimov Yu Ya and Naberukhin Yu I (2003) Fluctuation theory of hydrogen bonding applied to vibration spectra of HOD molecules in liquid water. I. Raman spectra. Mol Phys 101: 459-468

[18] Efimov Yu Ya and Naberukhin Yu I (2004a) Fluctuation theory of hydrogen bonding applied to vibration spectra of HOD molecules in liquid water. II. Infrared spectra: contour shape, integrated intensity, temperature dependence. Mol Phys 102: 1407-1414

[19] Efimov Yu Ya and Naberukhin Yu I (2004b) Thermodynamic functions of liquid water calculated from the temperature evolution of the vibration spectrum contour. Spectrochim Acta A 61: 1789-1794

[20] Brubach J-B, Mermet A, Filabozzi A, Gershel A and Roy P (2005) Signatures of the hydrogen bonding in the infrared bands of water. J Chem Phys 122: 184509 
[21] Bratos S, Leicknam J-C, and Pommeret S (2009) Relation between the OH-stretching frequency and the OO distance in time-resolved infrared spectroscopy of hydeogen bonding. Chem Phys 359: 53-57

[22] Nucci NV and Vanderkooi JM (2005) Temperature dependence of hydrogen bonding and freezing behavior of water in reverse micelles. J Phys Chem B 109: 18301-18309

[23] Crupi V, Longo F, Majolino D, and Venuti V (2007) Raman spectroscopy: probing dynamics of water molecules confined in nanopous silica glasses. Eur Phys J Special Topics 141: 61-64

[24] Maréchal Y (2007) The Hydrogen Bond and the Water Molecule. Elsevier

[25] Maréchal Y (1991) Infrared spectra of water. I. Effect of temperature and of H/D isotopic dilution. J Chem Phys 95: 5565-5573

[26] Libnau FO, Kvalheim OM, Christy AA and Toft J (1994a) Spectra of water in the near- and mid-infrared region. Vib Spectrosc 7: 243-254

[27] Czranik-Matusewicz B, Pilorz S, and Hawranek JP (2005) Temperature-dependent water structural transitions examined by near-IR and mid-IR spectra analyzed by multivariate curve resolution and two-dimensional correlation spectroscopy. Anal Chim Acta 544: 15-25

[28] Green JL, Lacey AR and Sceats MG (1986) Spectroscopic evidence for spatial correlations of hydrogen bonds in liquid water. J Phys Chem 90: 3958-3964

[29] Libnau FO, Toft J, Christy AA, and Kvalheim OM (1994b) Structure of liquid water determined from infrared temperature profiling and evolutionary curve resolution. J Am Chem Soc 116: 8311

[30] Stanley HE and Teixeira J (1980) Interpretation of the unusual behavior of $\mathrm{H}_{2} 0$ and $\mathrm{D}_{2} 0$ at low temperatures: Tests of a percolation model. J Chem Phys 73: 3404-3422

[31] Vand V and Senior W A (1965) Structure and partition Function of liquid water. III. Development of the partition function for a band model of water. J Chem Phys 43: 1869-1884

[32] Némethy G and Scheraga H A (1962) Structure of water and hydrophobic bonding in proteins. I. A model for the thermodynamic properties of liquid water. J Chem Phys 36: 3382-3400

[33] Eisenberg D and Kauzmann W (1969) The structure and properties of water. Clarendon Press, Oxford

[34] Buijs K and Chopin GR (1963) NearInfrared studies of the structure of water. I. Pure water. J Chem Phys 39: 2035-2045

[35] Buijs K and Chopin GR (1964) Assignment of the near-infrared bands of water and ionic solutions. J Chem Phys 40: 3120-3121 